\title{
CERVICAL TUBERCULAR LYMPHADENITIS:EVALUATION OF DIAGNOSTIC MODALITIES
}

Rajiv Jain, Ravi Dosi

1. Assistant Professor. Department of General Surgery, SAIMS, Indore.

2. Assistant Professor. Department of Respiratory Medicine, SAIMS, Indore.

\section{CORRESPONDING AUTHOR:}

Dr. Ravi Dosi,

Dosi Bhawan, 124, Tilak Nagar Main,

Post office road, Indore - 452018,

Madhya Pradesh, India.

E-mail: ravi.dosi@gmail.com

\begin{abstract}
The diagnosis of cervical tuberculous lymphadenitis has been performed by histological examination using excisional biopsy specimens. However a non-invasive diagnostic procedure alternative to invasive excisional biopsy has been required and fine needle aspiration cytology as well the polymerase chain reaction (PCR) techniques have become useful modalities. The aim of this study was to evaluate and compare different diagnostic modalities and establish their effectiveness in current conditions. We performed a retrospective study of two hundred patients of tuberculous cervical lymphadenitis admitted in the department of surgery, SAIMS Medical college and PG institute in a two year period from January 2011 to December 2012. Excision biopsy specimens and needle aspiration specimens were collected from all patients who were suspected as having cervical tuberculous lymphadenitis on the basis of clinical features, Mantoux test and other diagnostic techniques. The histopathological positive rates, the time period necessary for diagnosis, the rupture rate of the local skin lesion and the detection ratio of mycobacterium were compared between the two percutaneous approaches. The diagnosis of tuberculous lymphadenitis was successful in all cases either by cytological examination or with the histological approach. The sensitivity and the diagnostic efficacy of FNAC in detecting tubercular lymphadenitis were $87 \%$ and $80 \%$ respectively. The rupture rate of the local skin after the excisional biopsy was higher than that of the needle aspiration procedure. The time required for diagnosis was significantly longer than that of the needle aspiration procedure . Fine needle aspiration cytology is highly recommended as a less invasive method of diagnosis of cervical tuberculous lymphadenitis, but excision biopsy and histological examination is the gold standard.
\end{abstract}

INTRODUCTION: Cervical lymphadenitis is the most common head and neck manifestation of tubercular infections. The incidence of tubercular cervical lymphadenitis has increased either as a manifestation of a systemic tuberculous disease or a unique clinical entity localized to neck. It remains a diagnostic and therapeutic challenge because it mimics other pathologic processes and yields inconsistent physical and laboratory findings.

A high index of suspicion is needed for the diagnosis of tubercular cervical lymphadenitis. A unilateral single or multiple painless lump, mostly located in posterior cervical or supraclavicular region can occur. A thorough history and physical examination, tuberculin test, staining for acid-fast bacilli, radiologic examination, fine-needle aspiration and PCR will be very helpful in arriving at an early diagnosis and institution of treatment before a final diagnosis can be made by biopsy and culture. Tubercular lymphadenitis is best treated as a systemic 
disease with antitubercular medication. Treatment monitoring is more complex due to peculiar behavior of TB lymph nodes. Situation has become worse due to sharp increase in the incidence of atypical mycobacteria, poorly controlled HIV epidemic and rise of drug-resistant TB lymphadenitis. Surgical therapy along with antitubercular medication can be beneficial in selected patients.

MATERIALS AND METHODS: The duration of study was 2 years spanning from January 2011 to December 2012. We studied the case records of all the 550 patients admitted in the department of surgery for the palpable neck masses. out of those 550 cases, The patients who were having diagnosis other than tubercular cervical adenitis and the patients who did not complete treatment were excluded. Patients with bleeding diathesis and allergy to local anaesthetic were excluded.

200 patients who were confirmed as tubercular cervical lymphadenitis after full diagnostic workup and who undertook full prescribed treatment regimen were included in this retrospective study.

Follow up study of these patients were performed on basis of available contact details and data stored in the medical record department \& outpatient department.

All the patients were investigated on the lines of established investigation protocol.

Investigation protocol included routine hematological and biochemical blood tests , Fasting ESR , serum Adenosine Deaminase tests , Mantoux test and chest x-ray.

The patients underwent FNAC which was followed by surgical excision biopsy of the same lymph node.

Fine needle aspiration cytology was performed with 22 gauge needle attached to a $10 \mathrm{ml}$ syringe, without local anaesthesia.

Two smears were prepared from each patient .The alcohol fixed smears were stained with hematoxylin and eosin (H \& E). All slides were seen by consultant histopathologists at Department of surgical pathology, SAIMS.

Surgical excision biopsy was done under local anaesthesia. After infiltrating the area with 5 to $10 \mathrm{ml}$ of $1 \%$ lignocaine with 1:1000000 adrenaline, incision was made in skin crease over the lymph node. Lymph node was gently dissected free of surrounding tissue and hemostasis was secured. Wound was closed in two layers. The biopsy specimen was immediately fixed in $10 \%$ buffered formalin. The sample was processed in an automatic tissue processor for paraffin block preparation. From each block 2-3 micron thick sections were prepared by using rotatory microtome. All sections were then mounted on the slides and stained with routine $\mathrm{H}$ \& $\mathrm{E}$ staining methods. All slides were seen by consultant histopathologists at Department of surgical pathology, SAIMS.

There was no significant morbidity and mortality during diagnostic workup. After establishing diagnosis, all patients were given antitubercular chemotherapy.

RESULTS: The study was done in total 200 patients, out of which 124 [62\%] were male and $76[38 \%]$ were female.

The age range was from 15 to 72 years with a mean age of 24.14 years. 
Fine Needle Aspiration Cytology

\begin{tabular}{|l|l|l|}
\hline Pathology & Number & Percentage \\
\hline Tubercular & 156 & 78 \\
\hline Reactive & 24 & 12 \\
\hline Malignant & 17 & 8.5 \\
\hline Others & 3 & 1.5 \\
\hline Total & 200 & 100 \\
\hline
\end{tabular}

Excision biopsy

\begin{tabular}{|l|l|l|}
\hline Pathology & Number & Percentage \\
\hline Tubercular & 138 & 69 \\
\hline Reactive & 18 & 9 \\
\hline Malignant & 38 & 19 \\
\hline Others & 6 & 6 \\
\hline Total & 200 & 100 \\
\hline
\end{tabular}

COMPARITIVE ANALYSIS OF DIAGNOSTIC MODALITIES

\begin{tabular}{|c|c|c|c|}
\hline & \multicolumn{2}{|c|}{ excision biopsy } \\
\hline & & tubercular & non tubercular \\
\hline fine needle & tubercular [156] & 136 [tp] & $20[\mathrm{fp}]$ \\
\hline \multirow[t]{3}{*}{ aspiration cytology } & non tubercular[44] & $20[\mathrm{fn}]$ & $24[\mathrm{tn}]$ \\
\hline & & \multicolumn{2}{|c|}{ excision biopsy } \\
\hline & & malignant & non malignant \\
\hline fine needle & malignant [17] & 16 [tp] & $1[\mathrm{fp}]$ \\
\hline aspiration cytology & non malignant [183] & $22[\mathrm{fn}]$ & $161[\mathrm{tn}]$ \\
\hline
\end{tabular}

\section{A] TUBERCULOSIS}

\begin{tabular}{|l|l|l|}
\hline & FNAC & PERCENTAGE \\
\hline SENSITIVITY & 0.87 & 87.2 \\
\hline SPECIFICITY & 0.54 & 54.5 \\
\hline POSITIVE PREDICTIVE VALUE & 0.87 & 87.2 \\
\hline NEGATIVE PREDICTIVE VALUE & 0.54 & 54.5 \\
\hline DIAGNOSTIC EFFICACY & 0.80 & 80 \\
\hline
\end{tabular}

B] MALIGNANCY

\begin{tabular}{|l|l|l|}
\hline & FNAC & percentage \\
\hline sensitivity & 0.42 & 42.1 \\
\hline specificity & 0.99 & 99.3 \\
\hline positive predictive value & 0.94 & 94.1 \\
\hline negative predictive value & 0.87 & 87.9 \\
\hline diagnostic efficacy & 0.88 & 88.5 \\
\hline
\end{tabular}


MORBIDITY: Out of 200 patients who have undergone FNAC and excision biopsy of lymph node, 182 patients did not suffer from any significant morbidity. 18 patients suffered from procedure related untoward effects, majority of which were found after excision biopsy.

The relative frequency of occurrence was:

1. The rupture rate of the local skin lesion- $6[3 \%]$

2. Chronic tubercular sinus-3[1.5\%]

3. Non healing ulcer-2[1\%]

4. Haemorhhage and pain during procedure-4[2\%]

5. Numbness and paresthesia at the operative site-3[1.5\%]

\begin{tabular}{|l|l|l|}
\hline Morbidity & FNAC & Excision Biopsy \\
\hline Rupture of skin lesion & 1 & 6 \\
\hline Chronic sinus & - & 3 \\
\hline Non healing ulcer & - & 2 \\
\hline Haemorrhage and pain & 2 & 4 \\
\hline Numbness and paresthesia & - & 3 \\
\hline
\end{tabular}

MORTALITY: No, mortality during any procedure was observed.

Time period: Fine needle aspiration cytology - two days

Excision biopsy - 4 days

DISCUSSION: Tuberculosis is an important health problem and it is the most common cause of infectious disease affecting the lymphoid tissue. In a study conducted in Kathmandu, causes of cervical lymphadenopathy were tuberculous lymphadenitis (54\%), reactive hyperplasia (33\%) and metastatic lesion (11.1\%). FNAC was found to be highly effective (94\%) in diagnosis and lymph nodes in the posterior triangle of neck were mostly involved.[19] In our study tuberculosis was also the main cause of cervical lymphadenopathy[69\%] but is

relatively high as compared to study cited above.

A high index of suspicion is needed for the diagnosis of tubercular cervical lymphadenitis. The key to early diagnosis is a detailed history and physical examination, tuberculin test, staining for acid-fast bacilli, radiologic examination, and fine -needle aspiration cytology which will allow early institution of therapy before a final diagnosis can be made by biopsy and culture. [1,2]

The differential diagnosis is extensive and includes infections (viral, bacterial or fungal), and neoplasms (lymphoma or sarcoma, metastatic carcinoma), non-specific reactive hyperplasia, sarcoidosis, toxoplasmosis, cats-scratch fever, collagen vascular diseases and diseases of reticuloendothelial system.

FNAC is a simple, sensitive, specific and cost-effective way to diagnose tubercular cervical lymphadenitis [3] especially in children presenting with a suspicious neck swelling.[4] If cytological findings are inconclusive repeatedly, tissue biopsy by surgery is advisable $[5,6,7,8]$. The correlation of cytopathology and histopathology has already been discussed by several workers[15,16,17,18]

In our study, we evaluated the sensitivity, specificity, positive and negative predictive value of FNAC in diagnosing tubercular cervical lymphadenitis and malignant 
lymphadenopathy. We took the results confirmed by biopsy and histopathology as standard against which the results obtained by FNAC were compared. We found the sensitivity of FNAC in diagnosing Tubercular cervical lymphadenitis was $87 \%$ and specificity was $54 \%$.

Manitchotpisit et al had shown sensitivity and specificity of $48 \%$ and $87.5 \%$ respectively in the diagnosis of cervical tuberculosis on FNAC.[9]

Many studies have found FNAC as an highly sensitive and specific tool for the diagnosis of metastatic malignancies.

In our study 17 cases were diagnosed as having metastatic carcinoma on FNAC. 22 cases of metastatic carcinoma were missed on FNAC which were picked by histopathology.

Thus sensitivity and specificity of FNAC in detecting malignant lymph node was $42 \%$ and $99 \%$ respectively.

This was in sharp contrast to a similar study done by Shahzad Et al who found sensitivity to be $95 \%$ with $100 \%$ positive predictive value.[10]

Squamous cell carcinoma is the commonest malignancy presenting in the cervical lymph nodes, metastasizing from upper aero digestive tract a fact validated in our study.

Thus in our study we found that the sensitivity and the diagnostic efficacy of FNAC is very high in diagnosing tubercular cervical lymphadenitis. Though in our study the sensitivity of FNAC in diagnosing metastatic lymphadenopathy was low but the specificity was very high [99\%].so our study clearly highlights the diagnostic importance of FNAC in patients of cervical lymphadenopathy.

The relative sensitivities of different procedures and the potential therapeutic benefits should be considered in making the choice of diagnostic approach. Excisional biopsy is the most invasive approach to diagnosis; however, it has the highest sensitivity and may produce a more rapid and favorable symptomatic response [11] and has been recommended in cases involving multiple nodes [12]. Rare complications of biopsy include the rupture of the local skin lesion, postsurgical pain, wound infection, sinus formation, non healing ulcer and scar [13]. In a study from Hong Kong, $80 \%$ of specimens from excision biopsy yielded positive culture results, compared with $17 \%$ from fine-needle aspiration (FNA) specimens [14].

CONCLUSION: Histopathology is most rewarding for diagnosis of cervical lymphadenitis, its Feasibility is limited due to lack of facilities and non-acceptability, being an invasive procedure.

Previously, biopsy was used for diagnosis of tubercular lymphadenitis; now FNAC is greatly replacing it because of it being a noninvasive, simple and cost effective procedure which can easily be done in office or outpatient sitting.

\section{REFERENCES:}

1. Paredes C, Del Campo F, Zamarron C. Cardiac tamponade due to tuberculous mediastinal lymphadenitis. Tubercle. 1990;71:219-220.

2. Ibekwe AO, al Shareef Z, al Kindy S. Diagnostic problems of tuberculous cervical adenitis (scrofula). Am J Otolaryngol. 1997;18:202-205.

3. 3.Ellison E, Lapuerta P, Martin SE. Fine needle aspiration diagnosis of mycobacterial lymphadenitis. Sensitivity and predictive value in the United States. Acta Cytol. 1999;43:153-157.

4. Lau SK, Wei WI, Hsu C, Engzell UC. Fine needle aspiration biopsy of tuberculous cervical lymphadenopathy. Aust N Z J Surg. 1988;58:947-950. 
5. Dandapat MC, Mishra BM, Dash SP, Kar PK. Peripheral lymph node tuberculosis: a review of 80 cases. Br J Surg. 1990;77:911-912

6. Pang SC. Mycobacterial lymphadenitis in Western Australia. Tuber Lung Dis. 1992;73:362-367

7. Albright JT, Pransky SM. Nontuberculous mycobacterial infections of the head and neck. Pediatr Clin North Am. 2003;50:503-514.

8. Liu ES, Bernstein JM, Sculerati N, Wu HC. Fine needle aspiration biopsy of pediatric head and neck masses. Int J Pediatr Otorhinolaryngol. 2001;60:135-140.

9. Manitchotpisit B , Kunachak S,Kulapraditharom B, Sura T. Combined use offine needle aspiration cytology and polymerase chain reaction in the diagnosis of cervicaltuberculous lymphadenitis. J Med Assoc Thai 1999; 82:363-8.

10. Qasmi et al , Faran Kiani, Afaq Iqbal Malik, Jamil Salamatullah,Muhammad Omar Farooq, Muhammad Amjad Abassi J Sur Pakistan (International) 17 (2) April — June 2012

11. Artenstein AW, Kim JH, Williams WJ, Chung RC. Isolated peripheral tuberculous lymphadenitis in adults: current clinical and diagnostic issues. Clin Infect Dis 1995;20:87682.

12. Blaikley JF, Khalid S, Ormerod LP. Management of peripheral lymph node tuberculosis in routine practice: an unselected 10-year cohort. Int J Tuberc Lung Dis 2011;15:375-8.

13. Lee KC, Tami TA, Lalwani AK, Schecter G. Contemporary management of cervical tuberculosis. Laryngoscope 1992;102:60-4.

14. Lau SK, Wei WI, Hsu C, Engzell UC. Efficacy of fine needle aspiration cytology in the diagnosis of tuberculous cervical lymphadenopathy. J Laryngol Otol 1990;104:24-7.

15. Nagpal, B.L., Dhar, C.N., Singh, A. and Bahi, H.H. Evaluation of imprint cytodiagnosis in case of lymphadenopathyInd. J. P.M.; 1982, 25, 35.

16. Patra, A.K., Nanda, B.K., Mohaptra, B.K., and Panda, A.K. Diagnosis of lymphadenopathy by Fine Needle aspiration Cytology, IJPM; 1983, 26, 273.

17. Dandapat, M.C., Panda, B.K., Patra, A.K., and Acharya, N. Diagnosis of tubercular lymphadenitis by fine needle aspiration cytology. Ind. J. Tub.; 1987, 34,139.

18. Tripathy, S.N., Mishra, N., Patel, N.M.,Samantray, D.K., Das,B.K., and Mania, R.N.,Place of aspiration biopsy in the diagnosis of lymphadenopathy.Ind. J. Tub.; 1985, 32, 130.

19. Maharjan M, Hirachan S, Kafle PK, Bista M,Shrestha S, Toran KC, et al. Incidence of tuberculosis in enlarged neck nodes, our experience. Kathmandu Univ Med J 2009;7: 